\title{
RASCH: a new approach in tool validation
}

\author{
Dushyanthi Jayawardene $^{1 *}$, Nalika Gunawardena ${ }^{2}$, Madhuwanthi Dissanayake ${ }^{3}$ \\ ${ }^{1}$ Department of Community Medicine, Faculty of Medicine, University of Colombo, Sri Lanka; ${ }^{2}$ World Health \\ Organization Country Office for Sri Lanka, Colombo, Sri Lanka; ${ }^{3}$ Department of Anatomy, Faculty of Medicine, \\ University of Colombo, Sri Lanka \\ *Correspondence: dushyanthi@commed.cmb.ac.lk
}

DOI: http://doi.org/10.4038/jccpsl.v23i1.8100

Received on: 30 October 2016

Accepted on: 24 January 2017

\section{Introduction}

Validity of a tool is the extent to which it measures what it is expected to measure in the target population. There are many types of validity namely content, consensual, construct and criterion. When measuring health related quality of life (QoL), the most important type of validity that comes into play is construct validity (1). In construct validity, what is looked into is whether the tool measures only the intended construct and not any other closely related construct. In other words, the score obtained for a tool should only reflect the performance of the respondent to the intended construct (2). If the researcher assessing QoL is confident that all items in the tool targets only a single construct of QoL, confirmatory factor analysis alone is sufficient to assess the validity of the tool. However, often tools assessing QoL suffer the issue of not representing the construct in totality or face the threat of other sub dimensions creeping into the construct making the score either inappropriately too low or too high (3). It is in such instances that Rasch analysis is applied as the most appropriate method to assess construct validity of the tools assessing QoL (4).

Rasch analysis was developed by a Danish Mathematician who developed a Poisson model, which later came to be known as the Rasch model (5). Rasch analysis works upon the assumption that respondents with a higher level of achievement in QoL being measured would have a better score for any item, when compared to those with a lower level of achievement in QoL. The greatest advantage of Rasch Analysis is that it generates linear measures, which provide a true picture of achievement of QoL while showing the difference between individuals and groups (6). In Rasch analysis, the respondents of the tool and the items (questions in the tool) are placed on the same linear scale, ordered from the most able to the least able and from the most difficult to the least difficult, respectively (7).

The main aim in Rasch analysis is to assess whether the data fit the unidimensional Rasch model. There are many fit statistics that are used to assess whether or not the data fit the Rasch model. Unidimensional models occur when the observed responses to the items in the questionnaire correspond with the expected responses. The expectation is that easier tasks stated in the questionnaire would more likely be attempted by persons who are less able, and the more difficult tasks are more likely to be attempted by persons who are more able. The major assumption thus of Rasch model is that the probability of a person being able to carry out a task is solely a logistical function of the relative distance between the item location parameter and the respondent location parameter of the linear scales. Once model fit has occurred and the uni-dimensionality of the QoL tool is proved, data are transformed from the ordinal scale to the nominal scale and the unit of measurement becomes the logit (Log odds unit), which is the distance along the line of the variable, which increases the odds of observing the event by a factor of 2.718. Differential Item Functioning (DIF) could also be obtained following 
model fit. This gives us the probability of persons with the same ability being able to carry out similar tasks. Multi-dimensionality in other words, contamination with other constructs of the QoL tool is suspected when the items do not fit the Rasch model (5).

Rasch analysis could be carried out using several statistical software programs, all of which however need to be purchased. The "Vision related quality of life and visual ability assessment tool - the Sri Lankan version”, which has been developed to assess the vision related quality of life and visual ability among children aged 1115 years in the Colombo district is an example for a tool that was validated using Rasch analysis in Sri Lanka by the writers using Winsteps version 3.92.1.

\section{References}

1. Brown JD. What is construct validity? Shiken Research Bulletin 2000; 4(2): 8-12.

2. Abramson JH, Abramson ZH. Survey methods in community medicine. Edinburgh: Churchill Livingstone, 1999.

3. Messick S. Validity and washback in language testing. Language Testing 1996; 13(3): 241-256.

4. Kreiner S, Christensen KB. Item screening in graphical log linear Rasch models. Psychometrika 2011; 76: 228-256.

5. Tennant A, Stephen PM, Hagell P. Application of Rasch analysis in the development and application of quality of life instruments. Value in Health 2004; 7(1): 22-26.

6. Lamoureux E, Pesudovs K. Vision-specific quality-of-life research: a need to improve the quality. American Journal of Ophthalmology 2011; 151(2), 195-197.

7. Elliott DB, Pesudovs K, Mallinson T. Vision-related quality of life. Optometry and Vision Science 2007; 84(8): 656-658. 